\title{
Behaviors of Low-Resistivity Annular in the Front of Invasion Zone While Fresh-Water Mud Filtrate Invading into a Reservoir
}

\author{
Jianhua Zhang \\ Science College, Xi'an Shiyou University, Xi'an, 710065, China \\ jhzhang@xsyu.edu.cn
}

Keywords: Invasion; Low-resistivity annular; Fresh mud; Time

\begin{abstract}
The behaviors of low-resistivity annular in the front of invasion were studied using fluidflowing theory and petrophysics equations when fresh-water mud was used during drilling. For porous and permeable formations, mud cake permeability controls the invasion rate mainly. Greater values of formation porosity decreased the movement rate of the annular. Formation permeability hardly affects the behaviors of the annular. The present results provide physical understand for the low-resistivity annular.
\end{abstract}

\section{Introduction}

While drilling for petroleum exploration, the drilling mud was used. Driven by the pressure differential between the mud in the borehole and the native fluid in a porous and permeable formation, the mud filtrate will invade into the formation or reservoir. Thus, an invasion zone was formed [1].

The invasion process has significant effects on reservoir measurements using resistivity log tools. Hence, it is important to understand the characters of the invasion zone. The common mud used in site is fresh-water mud. When fresh mud invaded into a reservoir, a special phenomenon must be paid attention that is the low-resistivity annular in the front of invasion zone [2].

The low-resistivity annular results from the complexity of the invasion process, which not only relates to mud-filtrate properties, formation pressure differential and oil/water phase permeability; but the salinity difference between mud filtrate and formation water. In addition, the amount of mud filtrate pore into the reservoir will increased with invasion time. Hence, at various time stages, radial distributions of fluid and other parameters are different [3].

The present work calculated the behaviors of the low-resistivity annular in the front of invasion zone when some important parameters, such as mud-cake permeability, formation permeability and porosity, changed. The results could give profound understanding for this low-resistivity annular. Also, it is helpful for log analysts to obtain the information of invasion zone; furthermore, to evaluate reservoirs correctly.

\section{Fluid Flowing Equations}

During drilling, mud filtrate invades into a formation radially and displaces the native fluids in porous volumes. The process of displacement can be described using fluid flowing theory [4]. The hydrocarbon was displaced by mud filtrate immiscibly. During drilling, mud filtrate pours into the formation under the pressure differential between borehole and formation after the formation was opened. The subscript $w$ and $o$ was used to denote the water and oil phase respectively. Then, the invasion process is related to formation absolute permeability $k$, formation porosity $\varphi$, original saturations $S_{w}$ and $S_{o}$, pressure $P_{w}$ and $P_{o}$, fluid viscosities $\mu_{w}$ and $\mu_{o}$, fluid density $\rho_{w}$ and $\rho_{o}$, the mass flow rate per unit of rock volume $q_{w}$ and $q_{o}$, and so on. They satisfied the fluid flow equations at invasion time $t$ for radial displacement process:

$$
\frac{1}{r} \frac{\partial}{\partial r}\left(\frac{r \rho_{w} k k_{r w}}{\mu_{w}} \frac{\partial P_{w}}{\partial r}\right)+q_{w}=\frac{\partial}{\partial t}\left(\varphi \rho_{w} S_{w}\right)
$$




$$
\frac{1}{r} \frac{\partial}{\partial r}\left(\frac{r \rho_{o} k k_{r o}}{\mu_{o}} \frac{\partial P_{o}}{\partial r}\right)+q_{o}=\frac{\partial}{\partial t}\left(\varphi \rho_{o} S_{o}\right)
$$

Where, $r$ is the distance from the axis of the borehole. The saturation sum of oil phase $S_{o}$ and water phase $S_{w}$ must be unity, $S_{o}+S_{w}=1$, because the pores in formation are completely fluid filled.

If the radius of borehole is $r_{b}$ and the pressure in the borehole is $P_{\text {well }}$, the boundary conditions of (1) and (2) are:

$$
\left.P\right|_{r=r_{b}}=P_{w e l l},\left.\quad S_{w}\right|_{r=r_{b}}=1,\left.\quad \frac{\partial P}{\partial r}\right|_{r \rightarrow \infty}=0
$$

From Eq.(1) to Eq.(3), the pressures $P_{w}(r, t)$ and $P_{o}(r, t)$ and saturations $S_{w}(r, t)$ and $S_{o}(r, t)$ can be obtained as functions of invasion time $t$ and radial distance $r$. Flowing efficiency $\lambda$ for oil and water phase were introduced respectively as:

$$
\lambda_{o}=\frac{\rho_{o} k_{o} k_{r o}}{\mu_{o}}, \lambda_{w}=\frac{\rho_{w} k_{w} k_{r w}}{\mu_{w}}
$$

Thus, Eq.(1) and Eq.(2) can be rewritten as

$$
\begin{aligned}
& \frac{1}{r} \frac{\partial}{\partial r}\left(\lambda_{w} r \frac{\partial P_{w}}{\partial r}\right)+q_{w}=\frac{\partial}{\partial t}\left(\varphi \rho_{w} S_{w}\right) \\
& \frac{1}{r} \frac{\partial}{\partial r}\left(\lambda_{o} r \frac{\partial P_{o}}{\partial r}\right)+q_{o}=\frac{\partial}{\partial t}\left(\varphi \rho_{o} S_{o}\right)
\end{aligned}
$$

The differential form of Eq.(5) and Eq. (6) were numerically solved using a finite differential method as following:

$$
\begin{aligned}
& \frac{1}{r} \frac{\partial}{\partial r}\left(\lambda_{w} r \frac{\partial P_{w}}{\partial r}\right)=\frac{1}{r_{i} \Delta r_{i}}\left[\frac{2 \lambda_{w, i+1 / 2} r_{i+1 / 2}\left(P_{w, i+1}-P_{w, i}\right)}{\Delta r_{i+1}+\Delta r_{i}}-\frac{2 \lambda_{w, i-1 / 2} r_{i-1 / 2}\left(P_{w, i}-P_{w, i-1}\right)}{\Delta r_{i}+\Delta r_{i-1}}\right] \\
& \frac{1}{r} \frac{\partial}{\partial r}\left(\lambda_{o} r \frac{\partial P_{o}}{\partial r}\right)=\frac{1}{r_{i} \Delta r_{i}}\left[\frac{2 \lambda_{o, i+1 / 2} r_{i+1 / 2}\left(P_{o, i+1}-P_{o, i}\right)}{\Delta r_{i+1}+\Delta r_{i}}-\frac{2 \lambda_{o, i-1 / 2} r_{i-1 / 2}\left(P_{o, i}-P_{o, i-1}\right)}{\Delta r_{i}+\Delta r_{i-1}}\right]
\end{aligned}
$$

The subscription $i$ denotes $i$-th grid. When fresh-water mud was used during drilling, the mudfiltrate salinity $C_{m f}$ and native-water salinity $C_{w}$ is usually different. The mixing of mud filtrate with native water causes the variation of salinity distribution with time in the invasion process. The variation of water salinity is generated from dispersion equation [4]:

$$
\frac{1}{r} \frac{\partial}{\partial r}\left(\frac{r k k_{r w} C_{w}}{\mu_{w}} \frac{\partial P_{w}}{\partial r}\right)+q_{w} C_{m f}=\frac{\partial\left(\varphi S_{w} C_{w}\right)}{\partial t}
$$

with the boundary condition

$$
\left.C_{w}\right|_{r=r_{b}}=C_{m f}
$$

Eq. (8) can still be written in following finite differential form:

$$
\frac{\partial}{\partial r}\left(r \lambda C_{w} \frac{\partial P_{w}}{\partial r}\right)=\frac{2}{\Delta r_{i}}\left(\frac{\lambda_{w, i+1 / 2}^{n+1} C_{w, i+1 / 2}^{n+1}\left(P_{w, i+1}^{n+1}-P_{w, i}^{n+1}\right)}{\Delta r_{i+1}+\Delta r_{i}}-\frac{\lambda_{w, i-1 / 2}^{n+1} C_{w, i-1 / 2}^{n+1}\left(P_{w, i}^{n+1}-P_{w, i-1}^{n+1}\right)}{\Delta r_{i}+\Delta r_{i-1}}\right)
$$

A numerical method was programmed to solve Eqs.(6), (7) and (10). 
Petrophysics theory [5] is used to generate the formation water resistivity, $R_{w}(r, t)$, and formation resistivity, $R_{f}(r, t)$, as functions of time $t$ and radial coordinate $r$ for formation temperature $T\left({ }^{\circ} \mathrm{C}\right)$ :

$$
\begin{aligned}
& R_{w}(r, t)=\left(0.0123+\frac{3647.5}{C_{w}^{0.955}(r, t)}\right) \cdot \frac{82}{1.8 T+39} \\
& R_{f}(r, t)=\frac{a R_{w}(r, t)}{S_{w}^{n}(r, t) \varphi^{m}}
\end{aligned}
$$

They are determined from above-solved invasion profiles of saturation and salinity. Finally, Eq.(12) gives the resistivity profiles in formation when mud filtrate invaded.

\section{Behaviors of Low-Resistivity Annular}

When fresh-water mud invaded into a reservoir, a low-resistivity annular in the front of invasion zone was formed. Fig.1a illustrated the low-resistivity annular and its migration into the depth of formation at various time stages $(0,2,5,10$ and 17 days respectively) after the reservoir was reached. The horizontal axis denoted the distance from borehole and the vertical axis was the values of formation resistivity obtained from Eq.(12). The main input parameter in the present simulation calculation were: $C_{w}=80000 \mathrm{mg} / \mathrm{L}, C_{m f}=5000 \mathrm{mg} / \mathrm{L}, S_{w}=0.5, \varphi=0.1, k=0.1 \mu \mathrm{m}^{2}, \mu_{w}=4 \times 10^{-9} \mathrm{~Pa} \cdot \mathrm{s}$, $\mu_{o}=1 \times 10^{-7} \mathrm{~Pa} \cdot \mathrm{s}, \rho_{w}=1 \mathrm{~g} / \mathrm{cm}^{3}, \rho_{o}=0.7 \mathrm{~g} / \mathrm{cm}^{3}, T=90^{\circ} \mathrm{C}, m=2, n=2$, and $a=1$. Note that mud filtrate salinity is sixteen times lower than formation water.

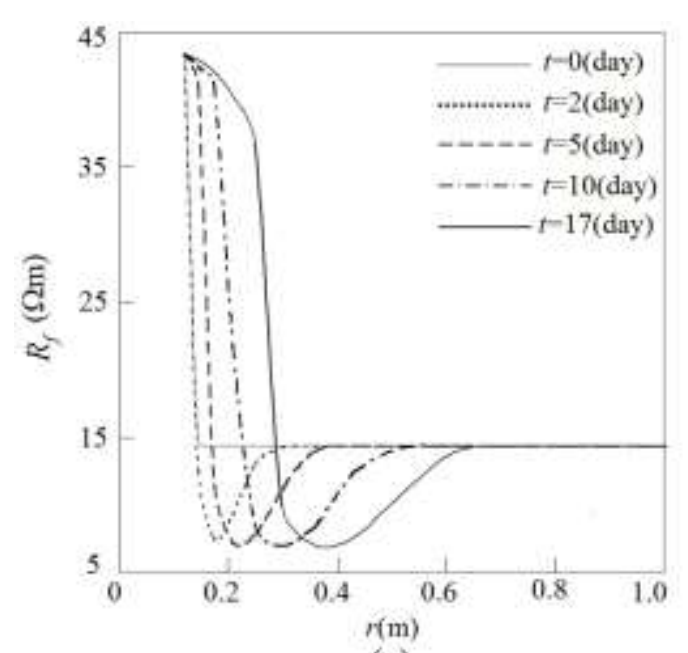

(a)

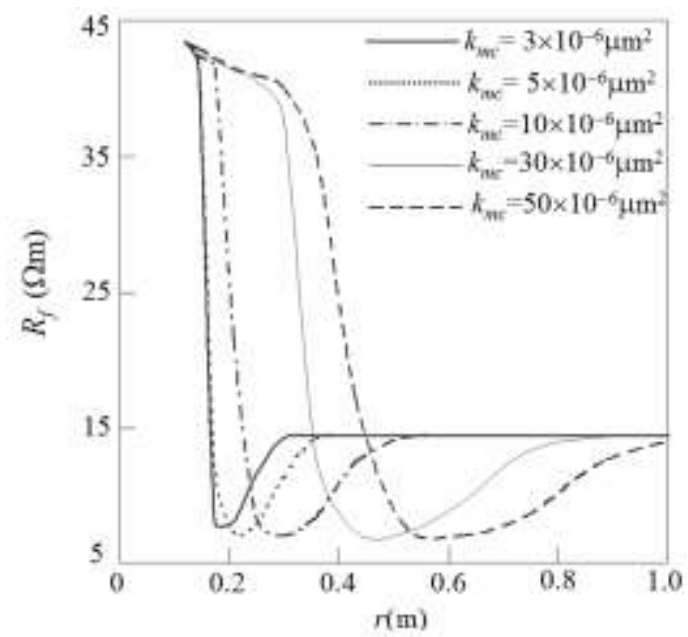

(b)

Figure 1. The low-resistivity annular in the front of invasion zone: (a) the annular variation with time $t$; (b) the annular behaviors with mud-cake permeability $k_{m c}$ at invasion time $t=5$ day

In the neighbor of borehole, the pores were filled with fresh-water mud filtrate, thus highresistivity mud filtrate. With the lapse of invasion, the high-resistivity mud filtrate pored into the formation, hence the profile of $R_{f}$ changed at various time stages, as shown in Fig.1(a). In addition, high water saturation and low mud salinity in the adjacent to the borehole caused a low resistivity range; that is the low-resistivity annular in the front of invasion zone.

Under the driving force of pressure difference between borehole and original formation, the mud filtrate moves to the formation gradually. At the beginning of bit penetration, mud poured into formation rapidly and mud cake is built up within a short time stage [5]. Mud cake controls the invasion rate mainly through its permeability $k_{m c}$. Fig.1(b) illustrated the effects of $k_{m c}$ on invasion zone and the low-resistivity annular at the invasion happening 5day for five different values of $k_{m c}$. The main input parameters are the same as in Fig.1(a). Greater $k_{m c}$ values mean rapid invasion rate. 
Both the invasion zone and low-resistivity annular were affected by formation porosity $\varphi$. Fig.2(a) illustrated the effects of $\varphi$. The main input parameters were the same as in Fig.1(a). According to Eq.(13), water resistivity, water saturation and porosity determined the formation resistivity. Formation resistivity decreased with increasing porosity $\varphi$. Greater $\varphi$ means that more mud filtrate was needed to fill the pores in reservoir, so that the invasion rate deceased and the lowresistivity annular is closer to the borehole, as shown in Fig.2(a).

According to Eq.(13), the formation resistivity does not relate to formation permeability $k$ directly; so that its profiles and the low-resistivity annular hardly change with various $k$, as shown in Fig.2(b).

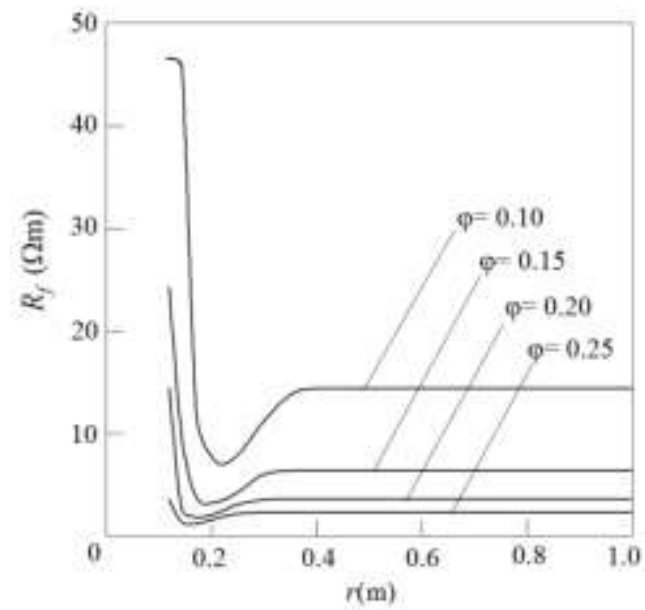

(a)

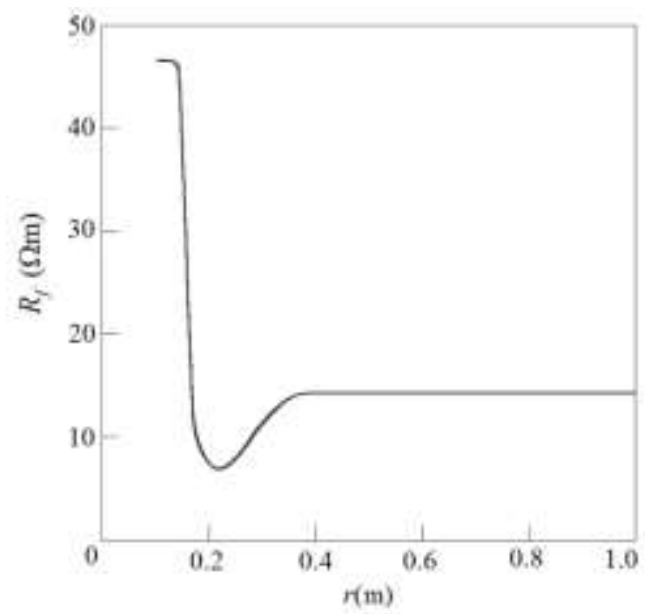

(b)

Figure 2. The low-resistivity annular behaviors with various porosity (a) and permeability (b) at invasion time $t=5$ day

\section{Conclusion}

While fresh-water mud was used during drilling, the low-resistivity annular in the front of invasion zone has important influence on the measurement of reservoirs after the formation was opened in petroleum exploration. Its migration can be solved from fluid-flowing equations.

Mud cake permeability $k_{m c}$ controls the invasion rate mainly; hence, greater $k_{m c}$ causes the lowresistivity annular to move into formation rapidly.

Greater values of porosity decreased the movement rate of the low-resistivity annular. Formation permeability hardly affects the behaviors of the low-resistivity annular.

The present study provided the physical understand for invasion process. Furthermore, they are helpful for following measurements of resistivity logging devices [5].

\section{References}

[1] J. Tittman, Geophysical Well Logging, Academic Press, Inc., Orlando, p.82, 1986

[2] A.Y. Windarto, P. Gunawan, E. Sukarno, Modeling of mud filtrate invasion and damage zone formation, J. Pet. Sci. Eng. Vol.77(3-4), p.359-364, 2011.

[3] Akinsete, Oluwatoyin O. and Adekoya, Doyinsola A., Effects of mud filtrate invasion on well log measurements, SPE Nigeria Ann. Inter. Conf. and Ex., August 2-4, 2016

[4] G.W.Thomas, Principles of Hydrocarbon Reservoir Simulation, International Human Resources Development Corporation, Boston, p.112, 1982

[5] J. H. Zhang. and Z. H. Liu, Time-dependant responses of high definition induction log and case studies, Chinese Journal of Engineering, Vol.2014, p.1-5, 2014 